\title{
A Sensor Fusion Approach for Localization with Cumulative Error Elimination
}

\author{
Feihu Zhang, Hauke Stähle, Guang Chen, Chao Chen \\ Carsten Simon, Christian Buckl and Alois Knoll
}

\begin{abstract}
This paper describes a robust approach which improves the precision of vehicle localization in complex urban environments by fusing data from GPS, gyroscope and velocity sensors. In this method, we apply Kalman filter to estimate the position of the vehicle. Compared with other fusion based localization approaches, we process the data in a public coordinate system, called Earth Centred Earth Fixed (ECEF) coordinates and eliminate the cumulative error by its statistics characteristics. The contribution is that it not only provides a sensor fusion framework to estimate the position of the vehicle, but also gives a mathematical solution to eliminate the cumulative error stems from the relative pose measurements (provided by the gyroscope and velocity sensors). The experiments exhibit the reliability and the feasibility of our approach in large scale environment.
\end{abstract}

\section{INTRODUCTION}

Within the past decade, accurate global localization has become a hot issue in the intelligent vehicle research domain, not only for developing advanced driver assistance system, but also for achieving autonomous driving.

Since GPS is susceptible to interference or even not fully available (tunnels, indoor environments, mountainous forested environments), odometry based localization is taken as a supplement solution to calculate the position of the vehicle by using e.g.vision based sensors (cameras, LIDARs, etc) [1], wheel sensors [2] and inertial sensors (gyroscopes and accelerometers) [3] [4]. However, as an odometry system without references to re-calibrate itself, the position will drift with the passage of time. Several methods have been demonstrated by using terrain maps [5] and visual landmark recognition [6] to reduce the cumulative error. However, in large scale urban environments, both require huge resources.

The idea of fusion based localization has been proposed in recent years to overcome this problem. Agrawal and Konolige [7] present a localization method using stereo vision and GPS. In their work, visual odometry is fused with GPS by using an extended Kalman filter. However, it requires that the GPS directly provides the trajectory in a local coordinates system (usually the GPS only provides the measurements as latitude, longitude and height in a global coordinate system). In addition, the extended Kalman filter which they used is suboptimal because of the non-linear transformations. Somphop et al. [8] utilize GPS, compass, wheel encoders with machine vision to improve the localization precision.

Feihu Zhang, Hauke Stähle, Guang Chen and Alois Knoll are with the Technische Universität München, Garching bei München, Germany, e-mail: feihu.zhang@tum.de, \{guang,staehle,knoll\}@in.tum.de.

Chao Chen, Carsten Simon and Christian Buckl are with the fortiss GmbH, München, e-mail: \{chen,simon,buckl\}@ fortiss.org.
Their machine vision technique extracts road signs and uses predefined map to calculate the vehicle's position. However, real time requirement is still an issue.

In this paper, we present a sensor fusion approach to integrate gyroscope, velocity sensor and GPS with the goal of improving the precision of localization in complex urban environments. The GPS provides the data in geodetic coordinates (Sec. II-A) while the others provide the data in navigation coordinates (Sec. II-A). Compared with others, we introduce a cumulative error elimination approach to improve the precision of the estimation. The experiments show that the integration scheme not only yields precise estimation but also satisfies the real-time requirement.

An off-the-shelf platform, OXTS RT 3000 GPS/IMU, is used to provide data under real traffic scenarios [9].

The remainder of this paper is structured as follows: Sec. II briefly describes different coordinates with their corresponding relationships. Sec. III introduces more details about the estimation phase. Sec. IV presents experimental results under different scenes. Finally, the paper is concluded in Sec.V.

\section{PREREQUISITES}

This section gives insights into the prerequisites, while the next section will elaborate on the estimation phase.

\section{A. Different coordinate systems}

In order to integrate different sensors for localization, several coordinates systems need to be introduced [10]:

1) Geodetic coordinate system: In geodetic coordinate system, the earth's surface is approximated by an ellipsoid and locations near the surface are described in terms of latitude $(\phi)$, longitude $(\lambda)$ and height $(h)$, which provide by the GPS. Fig. 1 shows the latitude and longitude of the vehicle in geodetic coordinates.

2) Earth-centred-earth-fixed (ECEF) coordinate system: The earth-centred-earth-fixed (ECEF) or conventional terrestrial coordinate system rotates with the earth and has its origin at the centred of the earth. The $X_{\text {ecef }}$ axis passes through the equator at the prime meridian. The $Z_{\text {ecef }}$ axis passes through the north pole but it does not exactly coincide with the instantaneous earth rotational axis. The $Y_{\text {ecef }}$ axis can be determined by the right-hand rule to be passing through the equator at 90 longitude. Fig. 1 shows the origin and three axis of the ECEF coordinate system. 


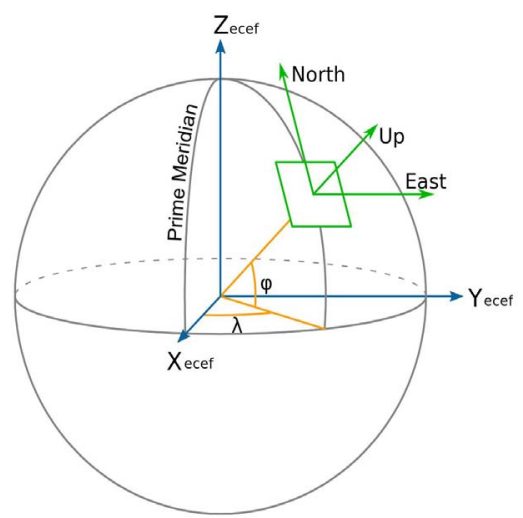

Fig. 1. Different coordinates systems

3) Local east, north, up (ENU) coordinate system: In many applications the local East, North, Up (ENU) Cartesian coordinate system (Fig. 1) is far more intuitive and practical than ECEF or geodetic coordinates. The local ENU coordinates are formed from a plane tangent to the earth's surface fixed to a specific location and hence it is sometimes known as a local tangent plane. By convention the east axis is labeled as $X$, the north $Y$ and the up $Z$.

4) Vehicle coordinate system: The vehicle coordinates $(x, y, z)$ are defined in a three-dimensional Cartesian coordinate system with its origin in the middle of the rear axle. The $\mathrm{x}$ axis and $\mathrm{y}$ axis are paralleled with the vehicle's horizontal axle and vertical axle while the $\mathrm{z}$ axis is perpendicular to the ground. The gyroscope and velocity sensors provide the egomotion vector $\left(\eta_{i}, d_{i}\right)$ at each frame, where $d$ is the velocity and $\eta$ is the rotation angle, $i$ is the frame index. (In this paper, we assume the vehicle is driving in a flat plane)

5) Navigation coordinate system: The navigation coordinates $\left(X_{\text {nav }}, Y_{\text {nav }}, Z_{\text {nav }}\right)$ are defined in a three-dimensional Cartesian coordinate system with origin on the initial position of the vehicle. It provides the whole trajectories by calculating the ego-motion vector as follows:

$$
X_{\text {nav }}=\sum_{i=1}^{n} d_{i} \sin \theta_{i} ; Y_{\text {nav }}=\sum_{i=1}^{n} d_{i} \cos \theta_{i} ; Z_{\text {nav }}=0
$$

where $\theta_{i}=\sum_{j=1}^{i} \eta_{j}$ is considered as the relative orientation in the navigation coordinate system, $i$ is the frame index.

Fig. 2 illustrates the relationship between the local ENU coordinates, the vehicle coordinates and the navigation coordinates. The $\mathrm{z}$ axis in the navigation coordinate system is equal to the corresponding axis in the others. However, the $y$ axis in the local ENU coordinates is parallel to the geodetic north compared with the corresponding axis.

\section{B. Coordinates conversion}

The transformations between the corresponding coordinates are as follows [10]:

1) Geodetic to ECEF: The geodetic coordinates can be converted into ECEF coordinates by using the following formulas:

$$
X_{\text {ecef }}=(N+h) \cos (\phi) \cos (\lambda)
$$

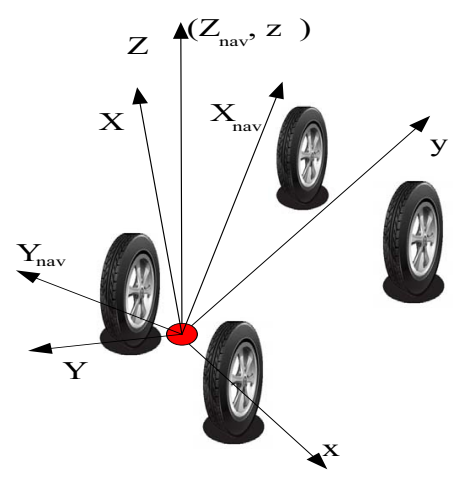

Fig. 2. Relationship between the local ENU coordinates, the vehicle coordinates and the navigation coordinates

$$
\begin{gathered}
Y_{\text {ecef }}=(N+h) \cos (\phi) \sin (\lambda) \\
Z_{\text {ecef }}=\left(N\left(1-e^{2}\right)+h\right) \sin (\phi)
\end{gathered}
$$

where

$$
N=\frac{a}{\sqrt{1-e^{2} \sin ^{2}(\phi)}}
$$

$e$ and $a$ denote eccentricity and the semi-major axis of the ellipsoid respectively.

2) ECEF to ENU: In order to transform the ECEF coordinates to the local ENU coordinates we need a local reference point, typically this might be the initial position of the vehicle $\left(X_{\text {ecef }}^{0}, Y_{\text {ecef }}^{0}, Z_{\text {ecef }}^{0}\right)$. Assuming this position is also the origin of the ENU coordinates, the related local ENU coordinates are calculated as follows

$$
\left[\begin{array}{c}
X \\
Y \\
Z
\end{array}\right]=A \times\left[\begin{array}{c}
X_{\text {ecef }}-X_{\text {ecef }}^{0} \\
Y_{\text {ecef }}-Y_{\text {ecef }}^{0} \\
Z_{\text {ecef }}-Z_{\text {ecef }}^{0}
\end{array}\right]
$$

where

$$
A=\left[\begin{array}{ccc}
-\sin \left(\lambda_{0}\right) & \cos \left(\lambda_{0}\right) & 0 \\
-\sin \left(\phi_{0}\right) \cos \left(\lambda_{0}\right) & -\sin \left(\phi_{0}\right) \sin \left(\lambda_{0}\right) & \cos \left(\phi_{0}\right) \\
\cos \left(\phi_{0}\right) \cos \left(\lambda_{0}\right) & \cos \left(\phi_{0}\right) \sin \left(\lambda_{0}\right) & \sin \left(\phi_{0}\right)
\end{array}\right]
$$

$\left(\phi_{0}, \lambda_{0}, h_{0}\right)$ is the geodetic coordinates of the origin $\left(X_{\text {ecef }}^{0}, Y_{\text {ecef }}^{0}, Z_{\text {ecef }}^{0}\right)$.

3) ECEF to Geodetic: Given ellipsoid $a, b$ and $f$, a approximation of geodetic coordinates is as follows:

$$
\begin{array}{r}
\phi=\tan ^{-1} \frac{Z_{e c e f}+e^{\prime} b \sin ^{3}(\theta)}{p-e^{2} a \cos ^{3}(\theta)} \\
\lambda=\tan ^{-1} \frac{Y_{e c e f}}{X_{e c e f}} \\
h=\frac{p}{\cos (\phi)}-N
\end{array}
$$


with

$$
\begin{array}{r}
b=a-a \cdot f \\
e^{\prime 2}=\left(a^{2}-b^{2}\right) / b^{2} \\
p=\sqrt{X_{\text {ecef }}^{2}+Y_{\text {ecef }}^{2}} \\
\theta=\tan ^{-1}\left(\frac{Z_{\text {ecef }} \cdot a}{p \cdot b}\right)
\end{array}
$$

where $b$ denotes the semi-minor axis of the ellipse, $a, N$ and $e$ are the same parameters in Sec. II-B.1.

More details about the integration process are given in Sec. III

\section{Estimating PHASE}

Since GPS is often susceptible in a large scale urban environment, odometry is taken as a supplement solution to localize the position of the vehicle. This phase not only introduces a fusion framework to provide the position estimation, but also gives a mathematical solution to eliminate the cumulative error which stems from the gyroscope and the velocity sensors.

\section{A. Sensor fusion process}

A sensor fusion solution is required since either GPS or odometry has its own limit. In this paper, we use the ECEF coordinate system to process the data from the whole sensors. The process is as follows:

First, the data from GPS is transformed from the geodetic coordinates to the ECEF coordinates.

Second, the data from gyroscope and velocity sensors is transformed form the vehicle coordinate system to the velocity in the local ENU coordinate system. In this step, the local ENU coordinate system is considered as a natural bridge to connect the vehicle coordinate system and the local ECEF coordinate system. The transformations are calculated as follows:

$$
\begin{array}{r}
\dot{X}_{i}=d_{i} \sin \gamma_{i} \\
\dot{Y}_{i}=d_{i} \cos \gamma_{i} \\
\dot{Z}_{i}=0 \\
\gamma_{i}=\theta_{i}+\delta
\end{array}
$$

where $i$ is the frame index, $\gamma_{i}$ and $\theta_{i}=\sum_{j=1}^{i} \eta_{j}$ are considered as the orientation in the ENU coordinate system and the relative orientation in the navigation coordinate system, $\delta$ is the rotation angle between the vehicle coordinate system and the ENU coordinate system on the origin of the ENU coordinates. $\left(\dot{X}_{i}, \dot{Y}_{i}, \dot{Z}_{i}\right)$ is the corresponding velocity in the local ENU coordinate system. Assuming the vehicle is driving on the flat plane, the velocity $\dot{Z}_{i}$ is considered as zero.

In addition, a Singular Value Decomposition (SVD) method [11] is used in this paper to calculate the rotation angle $\delta$ as follows:

Since the vehicle's trajectory can be calculated not only in the ENU coordinates (by Equ. (3)), but also in the navigation coordinates (by Equ. (1)). According to Euler's rotation theorem the transformation between the related two coordinates is as follows:

$$
\left[\begin{array}{c}
X_{n a v} \\
Y_{\text {nav }} \\
Z_{\text {nav }}
\end{array}\right]=\left[\begin{array}{ccc}
\cos \delta & -\sin \delta & 0 \\
\sin \delta & \cos \delta & 0 \\
0 & 0 & 1
\end{array}\right]\left[\begin{array}{c}
X \\
Y \\
Z
\end{array}\right]
$$

Based on Equ. (5), the SVD method calculates the rotation angle $\delta$ and the corresponding velocity in the ENU coordinates.

Third, according to Equ. (3), we have the following equations:

$$
\left[\begin{array}{c}
\dot{X} \\
\dot{Y} \\
\dot{Z}
\end{array}\right]=A \times\left[\begin{array}{c}
\dot{X}_{e c e f} \\
\dot{X}_{\text {ecef }} \\
\dot{X}_{\text {ecef }}
\end{array}\right]
$$

with

$A=\left[\begin{array}{ccc}-\sin \left(\lambda_{0}\right) & \cos \left(\lambda_{0}\right) & 0 \\ -\sin \left(\phi_{0}\right) \cos \left(\lambda_{0}\right) & -\sin \left(\phi_{0}\right) \sin \left(\lambda_{0}\right) & \cos \left(\phi_{0}\right) \\ \cos \left(\phi_{0}\right) \cos \left(\lambda_{0}\right) & \cos \left(\phi_{0}\right) \sin \left(\lambda_{0}\right) & \sin \left(\phi_{0}\right)\end{array}\right]$

Finally, the Kalman filter is given by:

$$
\begin{aligned}
\mathbf{t}_{k+1} & =\mathbf{F t}_{k}+\mathbf{l}_{k} \\
\mathbf{q}_{k+1} & =\mathbf{H}_{k+1} \mathbf{t}_{k+1}+\mathbf{v}_{k+1}
\end{aligned}
$$

with

$$
\begin{gathered}
\mathbf{t}=\left[\begin{array}{c}
X_{\text {ecef }} \\
Y_{\text {ecef }} \\
Z_{\text {ecef }} \\
\dot{X}_{\text {ecef }} \\
\dot{Y}_{\text {ecef }} \\
\dot{Z}_{\text {ecef }}
\end{array}\right], \mathbf{F}=\left[\begin{array}{llllll}
1 & 0 & 0 & 1 & 0 & 0 \\
0 & 1 & 0 & 0 & 1 & 0 \\
0 & 0 & 1 & 0 & 0 & 1 \\
0 & 0 & 0 & 1 & 0 & 0 \\
0 & 0 & 0 & 0 & 1 & 0 \\
0 & 0 & 0 & 0 & 0 & 1
\end{array}\right] \\
\mathbf{H}=\left[\begin{array}{cc}
\mathbf{I} & \mathbf{0} \\
\mathbf{0} & A
\end{array}\right]
\end{gathered}
$$

where $\mathbf{0}$ is a $3 \times 3$ zero matrix while $\mathbf{I}$ is a $3 \times 3$ identity matrix.

The state vector $\mathbf{t}$ describes the vehicle's position and its velocity in the ECEF coordinates, the measurement $\mathbf{q}$ is acquired by the first step and the second step. $\mathbf{l}_{k}$ and $\mathbf{v}_{k}$ are described as zero mean, normal distributed white noises.

According to the Kalman filter, the approach estimates the locations by using data from GPS, gyroscope and velocity sensors.

The Kalman filter also allows the localization in either GPS denied environment or odometry. It can be easily implemented by using the parameter $\mathbf{0}$ instead of $\mathbf{I}$ in observe matrix $\mathbf{H}$ when GPS fails. With the same manner, the parameter $A$ is replaced by $\mathbf{0}$ in observe matrix $\mathbf{H}$ when odometry also fails. 


\section{B. Cumulative error elimination}

We not only introduce a sensor fusion approach to estimate the location, but also give a mathematical solution to eliminate the cumulative error which provides by the odometry.

Assuming the ego-motion vector $\left(d_{i}, \eta_{i}\right)$ can be represented as

$$
\eta_{i}=\bar{\eta}_{i}+\tilde{\eta}_{i} ; d_{i}=\bar{d}_{i}+\tilde{d}_{i}
$$

where the errors $\tilde{\eta}_{n}$ and $\tilde{d}_{n}$ are assumed to be independent with zero mean and standard deviations $\delta_{\eta}$ and $\delta_{d}, \bar{\eta}_{n}$ and $\bar{d}_{n}$ are considered as the true value. The velocity of the vehicle in the local ENU coordinate system is calculated by the following equations:

$$
\begin{gathered}
\dot{X}_{i}=d_{i} \sin \gamma_{i}=d_{i} \sin \left(\sum_{j=1}^{i} \eta_{j}+\delta\right) \\
\dot{Y}_{i}=d_{i} \cos \gamma_{i}=d_{i} \cos \left(\sum_{j=1}^{i} \eta_{j}+\delta\right) \\
\dot{Z}_{i}=0
\end{gathered}
$$

The errors can also be represented by expanding

$$
\begin{aligned}
& \dot{X}_{i}=\dot{X}_{i}^{t}+\dot{X}_{i}^{e}=\left(\bar{d}_{i}+\tilde{d}_{i}\right) \sin \left(\sum_{j=1}^{i}\left(\bar{\eta}_{j}+\tilde{\eta}_{j}\right)+\delta\right) \\
& =\left[\bar{d}_{i}+\tilde{d}_{i}\right] \cdot\left\{\sin \left[\sum_{j=1}^{i} \bar{\eta}_{j}+\sum_{j=1}^{i} \tilde{\eta}_{j}\right] \cdot \cos \delta\right. \\
& \left.+\cos \left[\sum_{j=1}^{i} \bar{\eta}_{j}+\sum_{j=1}^{i} \tilde{\eta}_{j}\right] \cdot \sin \delta\right\} \\
& =\left[\bar{d}_{i}+\tilde{d}_{i}\right] \cdot\left\{\left[\sin \sum_{j=1}^{i} \bar{\eta}_{j} \cos \sum_{j=1}^{i} \tilde{\eta}_{j}+\cos \sum_{j=1}^{i} \bar{\eta}_{j} \sin \sum_{j=1}^{i} \tilde{\eta}_{j}\right]\right. \\
& \left.\cdot \cos \delta+\left[\cos \sum_{j=1}^{i} \bar{\eta}_{j} \cos \sum_{j=1}^{i} \tilde{\eta}_{j}-\sin \sum_{j=1}^{i} \bar{\eta}_{j} \sin \sum_{j=1}^{i} \tilde{\eta}_{j}\right] \sin \delta\right\}
\end{aligned}
$$$$
\dot{Y}_{i}=\dot{Y}_{i}^{t}+\dot{Y}_{i}^{e}=\left(\bar{d}_{i}+\tilde{d}_{i}\right) \cos \left(\sum_{j=1}^{i}\left(\bar{\eta}_{j}+\tilde{\eta}_{j}\right)+\delta\right)
$$$$
=\left[\bar{d}_{i}+\tilde{d}_{i}\right] \cdot\left\{\cos \left[\sum_{j=1}^{i} \bar{\eta}_{j}+\sum_{j=1}^{i} \tilde{\eta}_{j}\right] \cos \delta\right.
$$$$
\left.-\sin \left[\sum_{j=1}^{i} \bar{\eta}_{j}+\sum_{j=1}^{i} \tilde{\eta}_{j}\right] \sin \delta\right\}
$$$$
=\left[\bar{d}_{i}+\tilde{d}_{i}\right] \cdot\left\{\left[\cos \sum_{j=1}^{i} \bar{\eta}_{j} \cos \sum_{j=1}^{i} \tilde{\eta}_{j}-\sin \sum_{j=1}^{i} \bar{\eta}_{j} \sin \sum_{j=1}^{i} \tilde{\eta}_{j}\right]\right.
$$$$
\left.\cdot \cos \delta-\left[\sin \sum_{j=1}^{i} \bar{\eta}_{j} \cos \sum_{j=1}^{i} \tilde{\eta}_{j}+\cos \sum_{j=1}^{i} \bar{\eta}_{j} \sin \sum_{j=1}^{i} \tilde{\eta}_{j}\right] \sin \delta\right\}
$$

while $\dot{X}_{i}^{t}=\bar{d}_{i} \sin \left(\sum_{j=1}^{i} \bar{\eta}_{j}+\delta\right)$ and $\dot{Y}_{i}^{t}=\bar{d}_{i} \cos \left(\sum_{j=1}^{i} \bar{\eta}_{j}+\right.$ $\delta)$ are the true velocity of the vehicle, $\dot{X}_{n}^{e}, \dot{Y}_{n}^{e}$ are the corresponding errors. Based on the above equations we can obtain

$$
\begin{gathered}
\dot{X}_{i}^{e}=\bar{d}_{i}\left\{\left[\sin \sum_{j=1}^{i} \bar{\eta}_{j}\left(\cos \sum_{j=1}^{i} \tilde{\eta}_{j}-1\right)+\cos \sum_{j=1}^{i} \bar{\eta}_{j} \sin \sum_{j=1}^{i} \tilde{\eta}_{j}\right]\right. \\
\cdot \cos \delta+\left[\cos \sum_{j=1}^{i} \bar{\eta}_{j}\left(\cos \sum_{j=1}^{i} \tilde{\eta}_{j}-1\right)-\sin \sum_{j=1}^{i} \bar{\eta}_{j} \sin \sum_{j=1}^{i} \tilde{\eta}_{j}\right] \\
\cdot \sin \delta\}+\tilde{d}_{i}\left\{\left[\sin \sum_{j=1}^{i} \bar{\eta}_{j} \cos \sum_{j=1}^{i} \tilde{\eta}_{j}+\cos \sum_{j=1}^{i} \bar{\eta}_{j} \sin \sum_{j=1}^{i} \tilde{\eta}_{j}\right]\right. \\
\left.\cdot \cos \delta+\left[\cos \sum_{j=1}^{i} \bar{\eta}_{j} \cos \sum_{j=1}^{i} \tilde{\eta}_{j}-\sin \sum_{j=1}^{i} \bar{\eta}_{j} \sin \sum_{j=1}^{i} \tilde{\eta}_{j}\right] \sin \delta\right\} \\
\dot{Y}_{i}^{e}=\bar{d}_{i}\left\{\left[\cos \sum_{j=1}^{i} \bar{\eta}_{j}\left(\cos \sum_{j=1}^{i} \tilde{\eta}_{j}-1\right)-\sin \sum_{j=1}^{i} \bar{\eta}_{j} \sin \sum_{j=1}^{i} \tilde{\eta}_{j}\right]\right. \\
\cdot \cos \delta-\left[\sin \sum_{j=1}^{i} \bar{\eta}_{j}\left(\cos \sum_{j=1}^{i} \tilde{\eta}_{j}-1\right)+\cos \sum_{j=1}^{i} \bar{\eta}_{j} \sin \sum_{j=1}^{i} \tilde{\eta}_{j}\right] \\
\cdot \sin \delta\}+\tilde{d}_{i}\left\{\left[\cos \sum_{j=1}^{i} \bar{\eta}_{j} \cos \sum_{j=1}^{i} \tilde{\eta}_{j}-\sin \sum_{j=1}^{i} \bar{\eta}_{j} \sin \sum_{j=1}^{i} \tilde{\eta}_{j}\right]\right. \\
\left.\cdot \cos \delta-\left[\sin \sum_{j=1}^{i} \bar{\eta}_{j} \cos \sum_{j=1}^{i} \tilde{\eta}_{j}+\cos \sum_{j=1}^{i} \bar{\eta}_{j} \sin \sum_{j=1}^{i} \tilde{\eta}_{j}\right] \sin \delta\right\}
\end{gathered}
$$

Assuming $\tilde{\eta} \sim N\left(0, \delta_{\eta}^{2}\right)$ and $\tilde{d} \sim N\left(0, \delta_{d}^{2}\right)$, we can obtain

$$
\sum_{j=1}^{i} \tilde{\eta}_{j} \sim N\left(0, i \delta_{\eta}^{2}\right) ; \sum_{j=1}^{i} \tilde{d}_{i} \sim N\left(0, i \delta_{d}^{2}\right)
$$

In this case, we have

$$
\begin{gathered}
E\left(\cos \sum_{j=1}^{i} \tilde{\eta}_{j}\right)=e^{-i \frac{\delta_{\eta}^{2}}{2}} \\
E\left(\sin \sum_{j=1}^{i} \tilde{\eta}_{j}\right)=0
\end{gathered}
$$

The expectations of $\dot{X}_{i}^{e}$ and $\dot{Y}_{i}^{e}$ become

$$
\mu_{t}(\bar{\eta}, \bar{d})=\left[\begin{array}{c}
E\left[\dot{X}_{i}^{e} \mid \bar{\eta}, \bar{d}\right] \\
E\left[\dot{Y}_{i}^{e} \mid \bar{\eta}, \bar{d}\right]
\end{array}\right]
$$


where

$$
\begin{aligned}
& E\left[\dot{X}_{i}^{e} \mid \bar{\eta}, \bar{d}\right]=\bar{d}_{i}\left[\sin \sum_{j=1}^{i} \bar{\eta}_{j}\left(e^{-\frac{i \delta_{\eta}^{2}}{2}}-1\right) \cos \delta\right. \\
& \left.+\cos \sum_{j=1}^{i} \bar{\eta}_{j}\left(e^{-\frac{i \delta_{\eta}^{2}}{2}}-1\right) \sin \delta\right] \\
& E\left[\dot{Y}_{i}^{e} \mid \bar{\eta}, \bar{d}\right]=\bar{d}_{i}\left[\cos \sum_{j=1}^{i} \bar{\eta}_{j}\left(e^{-\frac{i \delta_{\eta}^{2}}{2}}-1\right) \cos \delta\right. \\
& \left.-\sin \sum_{j=1}^{i} \bar{\eta}_{j}\left(e^{-\frac{i \delta_{\eta}^{2}}{2}}-1\right) \sin \delta\right]
\end{aligned}
$$

Equation (10) is explicit expression for the expectation. The cumulative error has a significant bias, which depends on the knowledge of the true value. However, the expression cannot be used due to the fact that it is conditioned on the true value which is not available in practice. To make the result useful, the expected value of the true moment is evaluated conditioned on the measurement.

$$
E\left[\mu_{t} \mid \eta_{i}, d_{i}\right]=\mu_{i}
$$

Equation (11) is called the average true expectation. Expanding (11) using (8) then applying trigonometric identities, it can be shown that

$$
\begin{aligned}
& E\left[E\left(\dot{X}_{i}^{e}\right) \mid d_{i}, \eta_{i}\right] \\
& =E\left\{\overline { d } _ { i } \left[\sin \sum_{j=1}^{i} \bar{\eta}_{j}\left(e^{-\frac{i \delta_{\eta}^{2}}{2}}-1\right) \cos \delta\right.\right. \\
& \left.\left.+\cos \sum_{j=1}^{i} \bar{\eta}_{j}\left(e^{-\frac{i \delta_{\eta}^{2}}{2}}-1\right) \sin \delta\right]\right\} \\
& =E\left\{( d _ { i } - \tilde { d } _ { i } ) \left[\sin \sum_{j=1}^{i}\left(\eta_{j}-\tilde{\eta}_{j}\right)\left(e^{-\frac{i \delta_{\eta}^{2}}{2}}-1\right) \cos \delta\right.\right. \\
& \left.\left.+\cos \sum_{j=1}^{i}\left(\eta_{j}-\tilde{\eta}_{j}\right)\left(e^{-\frac{i \delta_{\eta}^{2}}{2}}-1\right) \sin \delta\right]\right\} \\
& =d_{i}\left(e^{-i \delta_{\eta}^{2}}-e^{\frac{-i \delta_{\eta}^{2}}{2}}\right)\left(\sin \sum_{j=1}^{i} \eta_{j} \cos \delta+\cos \sum_{j=1}^{i} \eta_{j} \sin \delta\right)
\end{aligned}
$$

With the same manner, the corresponding values of $\tilde{y}_{n}$ can be also represented as follows:

$$
\begin{aligned}
& E\left[E\left(\dot{Y}_{i}^{e}\right) \mid d_{i}, \eta_{i}\right]=d_{i}\left(e^{-i \delta_{\eta}^{2}}-e^{\frac{-i \delta_{\eta}^{2}}{2}}\right)\left(\cos \sum_{j=1}^{i} \eta_{j} \cos \delta\right. \\
& \left.-\sin \sum_{j=1}^{i} \eta_{j} \sin \delta\right)
\end{aligned}
$$

Finally, the velocity in the ENU coordinate system is acquired by the following equations:

$$
\begin{gathered}
{\left[\begin{array}{c}
\dot{X}_{i} \\
\dot{Y}_{i} \\
\dot{Z}_{i}
\end{array}\right]=} \\
{\left[\begin{array}{c}
d_{i} \sin \left(\sum_{j=1}^{i} \eta_{j}+\delta\right)-E\left[E\left(\dot{X}_{i}^{e}\right) \mid d_{i}, \eta_{i}\right] \\
d_{i} \cos \left(\sum_{j=1}^{i} \eta_{j}+\delta\right)-E\left[E\left(\dot{Y}_{i}^{e}\right) \mid d_{i}, \eta_{i}\right] \\
0
\end{array}\right]}
\end{gathered}
$$

After the cumulative error elimination, the estimation process is using the same Kalman filter to estimate the position of the vehicle.

\section{EXPERIMENTAL RESULTS AND DISCUSSION}

The vehicle platform is used from Karlsruhe dataset [9] which equipped with an OXTS RT 3000 GPS/IMU system (which provide the GPS data and the attitude of the vehicle, including the velocity and the rotation angle at each frame) with 10 frames/s. All sequences correspond to real traffic conditions in urban environments with pedestrians and cars. In the experiments, the vehicle was driven with an average velocity of $40 \mathrm{~km} / \mathrm{h}$. In this paper, the related measurements are acquired by manually adding the white noises on the original data. For GPS, the distribution of the measurement noise in geodetic coordinates is assumed with the deviation of $0.000075^{\circ}$ and $30 \mathrm{~m}$; for gyroscope, the measurement noise is considered as the deviation of $0.002 \mathrm{rad}$ while the velocity is considered as the deviation of $0.15 \mathrm{~m}$.

Fig. 3 illustrates the 2D trajectories acquired from the GPS/IMU system. The GPS measurements, the odometry (Equ. (1)) result, the sensor fusion approach without cumulative error elimination and the approach with error elimination are also included. Although the trajectories provided by GPS are not smoothing since the measurements' noise, however, the error is bounded compared with the odometry itself. From Fig. 3 we can see that our approach provides more reliable trajectories either with cumulative error elimination or without.

Table I also illustrates that the presented algorithm achieves high accuracy. The distance is the length of the experiments conducted while the RMSE (root mean square error) means the distance error between the estimated location and its true value. From table I we can see that the cumulative error elimination process improves the precision of the estimation compares with the other three methods.

The benefits of our approach are as follows:

First, it provides a sensor fusion framework to improve the precision of the global localization which utilizes the original data from GPS, gyroscope and velocity sensors. Second, it also eliminates the cumulative error stems from the odometry in large scale environment. Third, it can be easily implemented and satisfies the real time requirement in large scale urban environment. Forth, it estimates the location by using the Kalman filtering technique which provides the optimal estimation under Bayes framework. 


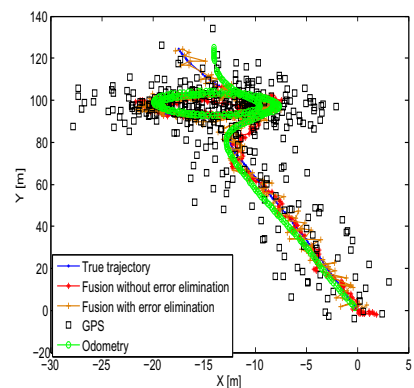

(a)

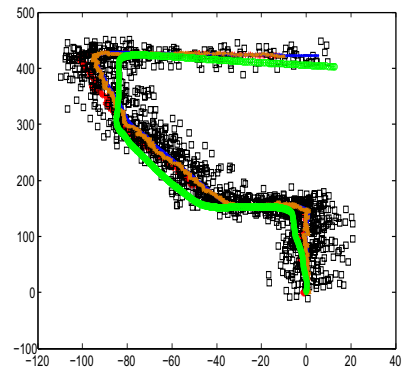

(b)

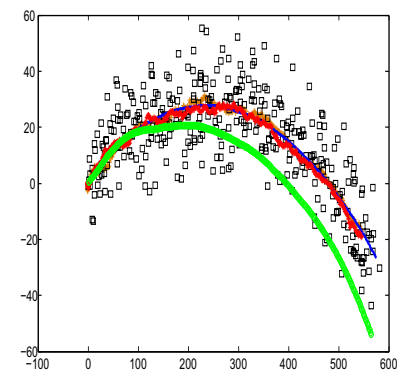

(c)

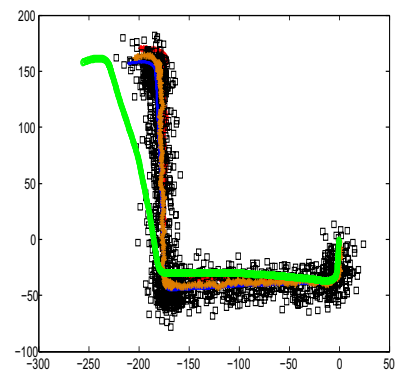

(d)

Fig. 3. Localization results

TABLE I

PERFORMANCE OF THE ALGORITHM

\begin{tabular}{l|l|l|l|l|l|l}
\hline Index & Distance & Frames & Odometry. RMSE & GPS. RMSE & $\begin{array}{l}\text { Fusion. RMSE } \\
\text { without error elimination }\end{array}$ & $\begin{array}{l}\text { Fusion.RMSE } \\
\text { with error elimination }\end{array}$ \\
\hline $\mathrm{a}$ & $310 \mathrm{~m}$ & 340 & $7.2161 \mathrm{~m}$ & $6.5768 \mathrm{~m}$ & $3.3127 \mathrm{~m}$ & $0.9362 \mathrm{~m}$ \\
\hline $\mathrm{b}$ & $640 \mathrm{~m}$ & 1249 & $15.0255 \mathrm{~m}$ & $13.3947 \mathrm{~m}$ & $9.9703 \mathrm{~m}$ & $6.3824 \mathrm{~m}$ \\
\hline $\mathrm{c}$ & $533 \mathrm{~m}$ & 355 & $16.7187 \mathrm{~m}$ & $13.2160 \mathrm{~m}$ & $3.0654 \mathrm{~m}$ & $3.0339 \mathrm{~m}$ \\
\hline $\mathrm{d}$ & $408 \mathrm{~m}$ & 1424 & $22.3397 \mathrm{~m}$ & $13.2044 \mathrm{~m}$ & $10.8963 \mathrm{~m}$ & $7.2029 \mathrm{~m}$ \\
\hline
\end{tabular}

The presented method has its own limit. A more precise model is required to represent the relative pose measurements. In this paper, we assume the noises are distributed as Gaussian distribution. However, in real urban environment, they are affected by various factors. A more completed mathematical model is therefore required.

\section{CONCLUSION}

Accurate global localization in urban environment is challenging in the present intelligent vehicle research domain. The GPS is susceptible to interference while the odometry often causes cumulative error. In this paper, a sensor fusion approach is presented to improve the localization precision in urban environment. In comparison to other works, we establish a public coordinates system to process data from the whole sensors by utilizing Kalman filtering technique. In addition, a cumulative error elimination process is also presented to improve the feasibility and reliability of the approach in large scale environment. The experiment shows that our approach improves the estimation precision compared with each individual sensor in complex urban environments.

Future improvements of the proposed approach include the use of other sensors such as radar and laser sensors in order to improve the estimation precision for automatic driving.

\section{REFERENCES}

[1] A. Milella and R. Siegwart, "Stereo-based ego-motion estimation using pixel tracking and iterative closest point," in IEEE International Conference on Computer Vision Systems, 2006 ICVS '06, Jan. 2006, p. 21.

[2] F. Chenavier and J. Crowley, "Position estimation for a mobile robot using vision and odometry," in Robotics and Automation, 1992. Proceedings., 1992 IEEE International Conference on, may 1992, pp. $2588-2593$ vol.3.
[3] T. Oskiper, Z. Zhu, S. Samarasekera, and R. Kumar, "Visual odometry system using multiple stereo cameras and inertial measurement unit," in Computer Vision and Pattern Recognition, 2007. CVPR '07. IEEE Conference on, june 2007, pp. $1-8$.

[4] J. Shen, D. Tick, and N. Gans, "Localization through fusion of discrete and continuous epipolar geometry with wheel and imu odometry," in American Control Conference (ACC), 2011, 29 2011-july 1 2011, pp. $1292-1298$.

[5] R. Lerner, E. Rivlin, and H. Rotstein, "Pose and motion recovery from feature correspondences and a digital terrain map," Pattern Analysis and Machine Intelligence, IEEE Transactions on, vol. 28, no. 9, pp. $1404-1417$, sept. 2006.

[6] M. Kais, S. Dauvillier, A. Foretllea, I. Masakid, and C. Laugier, "Towards outdoor localization using gis, vision system and stochastic error propagation," in International Conference on Autonomous Robots and Agents, 2004.

[7] M. Agrawal and K. Konolige, "Real-time localization in outdoor environments using stereo vision and inexpensive gps," in Pattern Recognition, 2006. ICPR 2006. 18th International Conference on, vol. 3, 0-0 2006, pp. $1063-1068$.

[8] S. Limsoonthrakul, M. Dailey, and M. Parnichkun, "Intelligent vehicle localization using gps, compass, and machine vision," in Intelligent Robots and Systems, 2009. IROS 2009. IEEE/RSJ International Conference on, oct. 2009, pp. $3981-3986$.

[9] B. Kitt, A. Geiger, and H. Lategahn, "Visual odometry based on stereo image sequences with ransac-based outlier rejection scheme," in Intelligent Vehicles Symposium (IV), 2010 IEEE, june 2010, pp. 486 -492 .

[10] Wikipedia, "Geodetic system - Wikipedia, the free encyclopedia," 2012, [accessed 06-March-2012]. [Online]. Available: http://en. wikipedia.org/wiki/Geodetic_system

[11] S. Umeyama, "Least-squares estimation of transformation parameters between two point patterns," Pattern Analysis and Machine Intelligence, IEEE Transactions on, vol. 13, no. 4, pp. 376 -380, apr 1991. 